\title{
Microtensile tests with the aid of probe microscopy for the study of MEMS materials
}

loannis Chasiotis, Wolfgang G. Knauss

loannis Chasiotis, Wolfgang G. Knauss, "Microtensile tests with the aid of probe microscopy for the study of MEMS materials," Proc. SPIE 4175, Materials and Device Characterization in Micromachining III, (11 August 2000); doi: 10.1117/12.395616

SDIE Event: Micromachining and Microfabrication, 2000, Santa Clara, CA, United States 


\title{
Microtensile Tests with the Aid of Probe Microscopy for the Study of MEMS Materials
}

\author{
Ioannis Chasiotis*, Wolfgang G. Knauss \\ Graduate Aeronautical Laboratories, California Institute of Technology, Pasadena, CA 91125
}

\begin{abstract}
Mechanical tests of thin films require novel and sophisticated methods that can address the geometry and microstructure of the films. A new method of micro-tensile testing of MicroElectroMechanical Systems (MEMS) films has been demonstrated. An improved apparatus has been designed and implemented to measure the elastic tensile properties (Young's modulus, Poisson's ratio and tensile strength) of surface micromachined polysilicon specimens. The tensile specimens are "dog-bone" shaped ending in a large "paddle" for convenient electrostatic or, in the improved apparatus, UV adhesive gripping. The test section of the specimens is $400 \mu \mathrm{m}$ long with $2 \mu \mathrm{m} \times 50 \mu \mathrm{m}$ cross section. The method employs Atomic Force Microscope (AFM) acquired surface topologies of deforming specimens to determine (fields of) strains by way of the Digital Image Correlation method (DIC). With this method, high strength or non-linearly behaving materials under different environmental conditions can be tested by measuring the strains directly on the surface of the film with nanometer resolution in in-plane and out-of-plane measurements.
\end{abstract}

Keywords: MEMS, polysilicon, tensile test, mechanical properties, UV adhesive grip, Atomic Force Microscopy, Digital Image Correlation, surface roughness

\section{INTRODUCTION}

During the last decade, numerous MEMS applications with significant commercial impact have been demonstrated and developed in the form of sensors or actuators. ${ }^{[1-4]}$ The reliability of MEMS devices is a major issue and it can only be addressed by direct measurements on small specimens with dimensions on the same order of magnitude as the fabricated microdevices. The film properties depend on the deposition conditions and the post-processing procedures and they are idiosyncratic for every clean room. There are several aspects of material properties that have to be addressed when developing a mechanical properties test method for MEMS devices. The method has to be flexible to provide measurements for different mechanical properties of interest. It is important that the method possess the capability of testing structural materials other than polycrystalline silicon that are considerably stronger such as $\mathrm{SiC}, \mathrm{Si}_{3} \mathrm{~N}_{4}$ and amorphous diamond and materials that behave non-linearly such as $\mathrm{Au}, \mathrm{Cu}$ or $\mathrm{Al}$ used for interconnects, or non-linear materials that are used as structural layers such as $\mathrm{Ni}$ in the LIGA process, or even viscoelastic materials such as polyimide or other rubber-like materials that the micromechanics community considers as candidates for future applications. Polycrystalline silicon is a superb material for the current applications but the investigation of new MEMS devices indicates the need for new application-specific materials. The constitutive behavior of those new materials is important to be measured without the use of any assumptions that are often needed in the use of indirect strain measurements. In addition to the constitutive behavior, other important quantities that are either material properties, such as the critical stress intensity factor of cracks or design and geometry related effects such as stress concentrations at notches and corners, with dimensions comparable to the grain size, need to be studied. The latter are strictly local in nature and demand the use of direct measurements on the surface of the film at the area of interest and cannot be addressed by the available strain measurements methods. Moreover, the test method has to possess the capability of conducting measurements in different environments such as in vacuum, in air, in the presence of inert gases, in extremely high ambient humidity or at high temperatures. It is important to develop a single and reliable method that addresses all the above since the use of different approaches for each one of the aforementioned conditions may result in ambiguous data as has been demonstrated by previous round robin studies 5 .

${ }^{*}$ Correspondence: Email:ichasiot@caltech.edu; WWW: http://www.its.caltech.edu/ ichasiot;Telephone: 626-395-4744; Fax: 626-449-2677

In Materials and Device Characterization in Micromachining III, Yuli Vladimirsky, Philip J. Coane, Editors, 
Finally, it is critical that the test method be able to address the characteristic length scale of the material at the scale of MEMS and take into account its microstructure, i.e. the grain size and the surface roughness.

Various techniques have been developed in the past to address the issues of mechanical integrity and evaluation of the elastic properties of polysilicon. Methods such as load-deflection, beam bending, tensile and nanoidentation tests or resonant frequency measurements of microcantilever beams have provided a span of values for the elastic constants. An important reason for the variety of reported results is the considerable difference in the test method, imprecise specimen geometries as well as the physical differences in the tested material. Of the aforementioned techniques, tensile tests ${ }^{5-12}$ are less vulnerable to geometry-induced errors and the measurements are easier to interpret from an error analysis point of view. The design and implementation of the tensile test apparatus can be complicated but the data interpretation is effortless and accurate. Uncertainties of the specimen thickness, density, undercuts, rigid body rotation at the specimen support, effect of the residual stress gradient in the actual deflection and the interpretation of the influence of those parameters on the experimental data are not an issue or can be incorporated in the data processing easier in tensile tests than in other methods. Additional parameters, such as surface roughness, can induce large uncertainties in the evaluation of test data. Numerical analysis ${ }^{6}$ proves, for instance, that the error due to the surface roughness in bending tests can be very important, sometimes exceeding the experimental and systematic errors while its effect on tensile test data is minimal. Tensile tests have been performed using electrostatics to grip the specimens, ${ }^{10}$ adhesive media ${ }^{9}$ or micromanipulators ${ }^{8}$ with specially designed specimens, either in a scanning electron microscope (SEM) ${ }^{8-10}$ or interferometrically ${ }^{7}$ to measure the displacements. In the present technique, direct measurements of the specimen surface can be obtained with sub-nanometer resolution by an AFM. A new and improved tensile tester is detailed along with a method capable of measuring strains locally on the surface of the specimen and results of modulus and strength of polysilicon films are presented.

\section{INSTRUMENTATION FOR MICROTENSILE TESTING}

An important aspect of the design process of the experimental apparatus for MEMS testing is the inherent dependence of the specimen geometry on the test device layout. The test method needs to be optimized taking into account the combination of optimum specimen and apparatus design. The experimental arrangement for subjecting small "dog-bone" shaped specimens to tension is shown in figure 1. The displacements are imposed to the specimen via an inchworm actuator that is powered by a system of a personal computer and a dedicated controller. The controller provides a measurement of the system (specimen + loading device) composite displacement with an accuracy of $4 \mathrm{~nm}$ for every single step of the actuator whose displacement resolution is $10 \mathrm{~nm}$. The induced load is measured by a miniature tension/compression load cell with an accuracy of $10^{-4} \mathrm{~N}$ and maximum capacity of $0.5 \mathrm{~N}$. A miniature $\mathrm{y}-\mathrm{z}$ translation stage is employed for sample positioning, while the setup allows for rotational adjustments about the $\mathrm{x}$-axis (along the load cell - specimen - inchworm axis) and $\mathrm{y}$-axis (on the plane of the specimen). The local deformation is monitored directly on the specimen surface by means of an AFM.

Experimenting with small specimens demands the test device to be designed so that it simultaneously addresses several important issues, as:

1. specimen mounting,

2. facilitation of the necessary electrical connections,

3. load cell, specimen and grip alignment, and

4. positioning accommodation under the AFM.

The performance of each individual component has been examined for satisfactory accuracy. The hysterisis and linearity of the load cell have been evaluated to identify possible sources of error. The linearity of the load-extension curve is very good for our purpose (error $<0.10 \%$ ). The effective value of hysterisis is on the order of $1.8 \%$ of the maximum load, restricted only to the low load region, and it is constant and consistent over a series of measurements. The resolution of the y-z translation stage is on the order of 1 micron; this is sufficient so that proper positioning of the grip can be achieved in the close proximity of the specimen surface before applying the voltage, or curing the adhesive. A horizontally positioned 300x optical microscope provides a side view of the tested beam during positioning. This helps preventing the undesirable specimen bending and facilitates monitoring of the specimen during the test. Due to the formation of a native oxide on the order of $2 \mathrm{~nm}$ on the surface of the specimen (the test is conducted in open air) the AFM is operated in Non-Contact Mode (NCM). The latter minimizes the effect of surface charging and cantilever tip induced vibration of the freestanding film during the measurement. 
The tensile tester was originally designed to take advantage of the electrostatic forces that can be exerted between two conductive surfaces separated by a non-conductive medium. The grip was comprised of a narrow silicon beam coated with a $200 \mathrm{~nm} \mathrm{Si}_{3} \mathrm{~N}_{4}$ insulating layer. The very thin electrical insulation layer made the necessary operational voltage relatively low but still close to the breakdown limits of the insulator. The operating voltage was on the order of $80 \mathrm{~V}$. This method has been used for most of the data presented in this work. An electrostatic grip however presents a maximum allowable induced load capability for a certain paddle size-insulator thickness combination, limited by the friction coefficient between the paddle and the electrostatic grip. The applied voltage, in conjunction with the local breakdown, can induce a charge on the surface of the specimen obstructing the use of a surface probing technique (AFM, STM). In addition to that, breakdown voltage and charging effects that occur due to the applied high voltage can easily cause severe damage to the test samples. Moreover, the recent trend to use materials other than polysilicon that have higher strength $\left(\mathrm{SiC}, \mathrm{Si}_{3} \mathrm{~N}_{4}\right)$ and better functionality in harsh environments, such as critical space applications, indicated the need to redesign the tensile test device. A new, more efficient gripping method has been developed that makes use of a high viscosity Ultraviolet (UV) adhesive. This newly developed tensile tester is free of any side-effects due to accumulated charge and facilitates the test of higher strength, or non-linearly behaving materials, making the proposed approach a universal test method for different thin films that do not even need to be conductive.

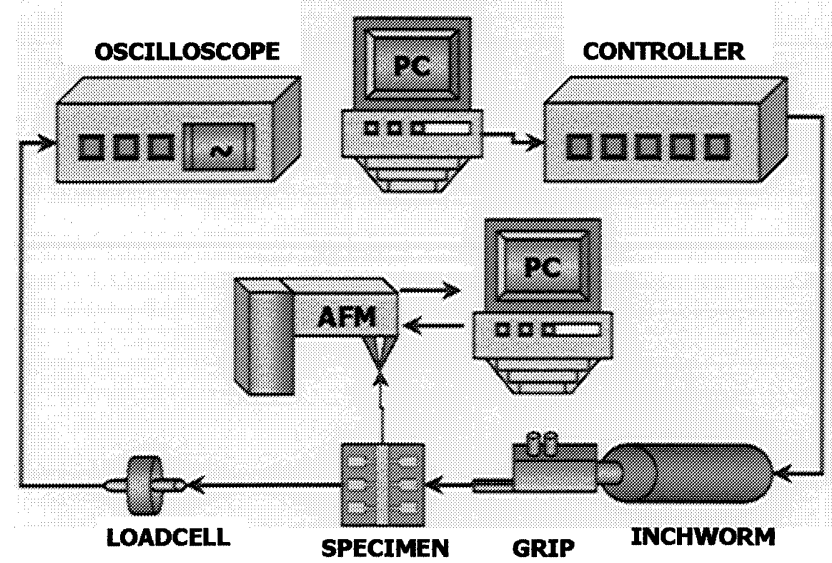

Figure 1. Left: Outline of the major components of the experimental setup. The strain can be measured either by using the closed loop inchworm actuator or directly on the surface of the specimen using the AFM. Right: Successive steps of film gripping: I. The film is forced electrostatically to lie flat on the substrate if it is bent due to the residual stress gradient, II. The glass grip with a UV curable adhesive layer is approached to the surface of the film, III. The same charge is induced to the substrate and the film to overcome any stiction forces by repelling the film from the substrate and forcing it to adhere, due to the exerted capillary forces, to the UV adhesive under the grip.

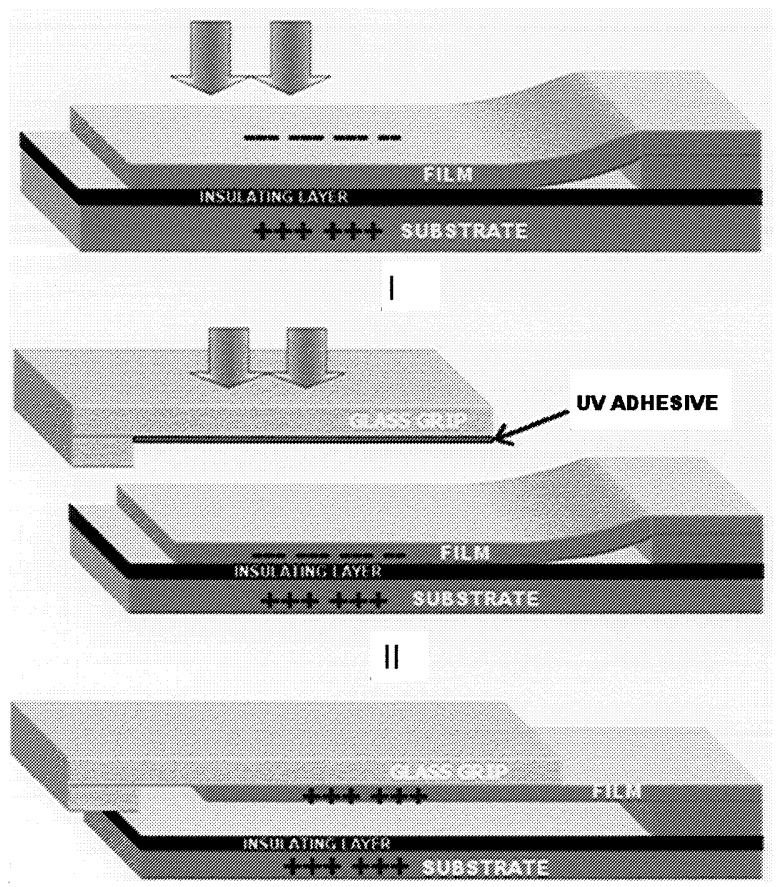

III

The operation of the newly implemented grip is illustrated in figure 1 on the right. Electrostatic forces are applied at first (figure 1 right, I) to force the thin polysilicon film to lie flat on the substrate (due to the effect of the residual stress gradient the released film it may be curved in the out-of-plane direction, causing buckling if gripped as is). The glass grip is then approached (figure 1 right, II) to the flat paddle. The grip is transparent to allow UV light to pass through and cure the underlying UV adhesive layer effectively and in minimal time. Next, the two surfaces of the substrate and the specimen paddle which adhere electrostatically and due to any stiction forces, are separated by providing both with the same charge so that the film is repelled by the substrate (figure 1 right, III) and adheres to the grip covered by a thin UV adhesive layer. Capillary forces keep the paddle in contact with the glass grip until the glue is cured by a short exposure to $365 \mathrm{~nm}$ UV light. The aforementioned process eliminates the need of mechanically pressing the grip against the paddle and the substrate, the potential flow of the adhesive on the substrate and consequently pre-test specimen damage. It is also important to note that the new grip provided us with nearly $100 \%$ rate of successful tests, increasing by a factor of two the rate of successful tests by means of the electrostatic grip. 
Digital Image Correlation (DIC) ${ }^{13-14}$ is the AFM data processing method used to determine the strain field. This method extracts in-plane deformations and resolves shape changes by comparing (surface) records before and after deformation. The DIC method makes use of the natural surface roughness features as being distributed markers. This information is obtained by a probe microscope (AFM) through records of surface features taken before and after deformation and then computing from this information the movement of the (three-dimensional) surface features. The method is capable of detecting displacements with a resolution of about $1 / 8$ of a pixel, which implies the capability of resolving strains on the order $0.03 \%$ $0.05 \%$ for a $512 \times 512$ data pixel AFM scan. For a detailed description of the method the reader is encouraged to refer to the listed references.

\section{SPECIMEN DESIGN AND CHARACTERISTICS}

Tensile, "bone-shaped" specimens were designed with test section dimensions of (LxWxD) 400x50x2 microns (figure 2), attached to the silicon die. Each die holds six freestanding specimens ending in a large paddle for convenient gripping. The specimens were designed for the Multi-User MEMS Processes (MUMPs)21 run at the Microelectronics Center of North Carolina (MCNC). The paddle is restricted from free movement by a tether attached at its free end. The tether is necessary due to the large dimensions of the paddle that make it extremely fragile during the wet release steps. It is also observed that the tethers prevent the paddles from completely adhering to the substrate and facilitate probing and releasing from stiction forces after storage. A custom probe stage has been built to cut the narrow beam that serves as an anchor for the paddle. The MUMPs21 specimens were designed so as to take into account the spatially frequent use of etch holes that facilitate the postprocessing stage of wet etching. The pattern for the paddle etch-holes was chosen to avoid specimen failure due to stress concentrations in the transition from the paddle to the test section if tested using the electrostatic grip. The specimen fabrication process obeys a three-polysilicon structural layer process and has been analytically described elsewhere ${ }^{6}$.

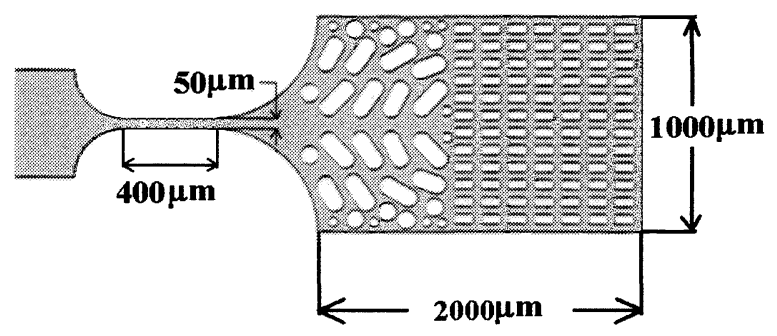

Figure 2. Dimensions of the designed tensile specimens. The exact thickness of the specimen is $1.9 \mu \mathrm{m}$.

The specimens were released at MCNC (now Cronos) using 49\% HF wet etch for 13 minutes with subsequent 15 minute water rinse and use of the supercritical $\mathrm{CO}_{2}$ method for drying in order to minimize the release stiction.

As we reported for previous MUMPS runs ${ }^{6}$ the fractured cross section of the specimens displayed a fairly columnar grain structure according to SEM cross-sectional images. An average grain size of $290 \mathrm{~nm}$ with a Gaussian distribution from $200 \mathrm{~nm}$ to $400 \mathrm{~nm}$ was measured via AFM. However finer structure, superimposed on the grains with characteristic lateral dimensions on the order of $50 \mathrm{~nm}$, has been observed via SEM. Measuring the polysilicon film thickness variation using an AFM, the ratio of the surface roughness to the specimen thickness is on the order of 1-2.5\%. This surface roughness has been identified as an important reason for the scatter of the results in the strength measurements when fracture is assumed to initiate at surface defects ${ }^{8}$. The polysilicon layer thickness was $1915 \mathrm{~nm}$ with a std. deviation $40 \mathrm{~nm}$, which corresponds to the average surface roughness. The two independent measurements (by means of AFM and SEM) provided almost the same results that were used to obtain more accurate values of the elastic constants instead of using the nominal value $(2 \mu \mathrm{m})$ for the film thickness. For example, the use of the nominal value of the thickness for the case of tensile test yields a $5 \%$ error that would translate into a $12 \%$ error for bending. 


\section{MICROTENSILE TESTS - RESULTS}

The specimens were gently probed before testing to free the paddle from being in contact with the substrate due to adhesion. ${ }^{15-16}$ Stiction forces were strong enough to overcome the action of the elastic restoring forces and thus the beams were not free on the substrate. Mechanical probing showed that the stiction forces were considerably lighter than observed for specimens that were not dried using the $\mathrm{CO}_{2}$ process. The light stiction forces can be attributed mainly to van der Waals, solid bridging and hydrogen bonding forces but not capillary forces which were eliminated by the supercritical $\mathrm{CO}_{2}$ process. The structures were mechanically probed with a fine and compliant tool. The tip was manufactured using the technique to fabricate sharp tips for STM devices by subjecting a $100 \mu \mathrm{m}$ in diameter tungsten wire to $\mathrm{NaOH}$ solution and shaping it electrochemically.

The loading device stiffness was measured at the end of the test and it was mostly comprised of the load cell stiffness. The contribution of the rest parts of the test system to the overall compliance was minimal. The test results were evaluated using ABAQUS to model the specimen geometry and the boundary conditions. Special consideration was given to other details of the specimen configuration such as undercuts and exact grip location. The experimental data, in conjunction with the finite element analysis, provided a value of the elastic modulus as $165.7 \pm 4.7 \mathrm{GPa}$, the value for the fracture strength as $1.00 \pm 0.1 \mathrm{GPa}$ and the maximum tensile strain as $0.65 \%$. The use of the electrostatic tensile tester provides us with an additional measurement. Based on the measured force values, for which relative slip between the grip and the paddle occurred, the static friction coefficient was calculated. As seen in table 1 the friction coefficient ranges from $0.07-0.1$ with an average of 0.08 . The scatter is attributed to the large variation of the surface roughness.

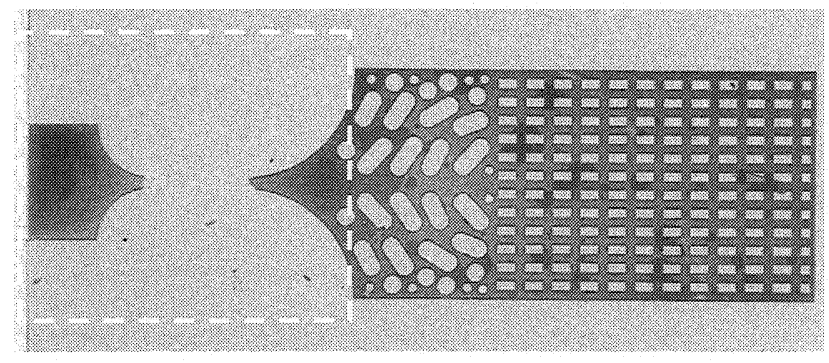

Figure 3. Left: Tested specimen indicating the dynamic nature of the fracture evolution after the onset of failure. The dashed line marked area is simulated. Right: Comparison of the marked specimen area with the modeled structure. Note that there are stress concentrations with $\mathrm{K}=1.04$ at the circled areas.

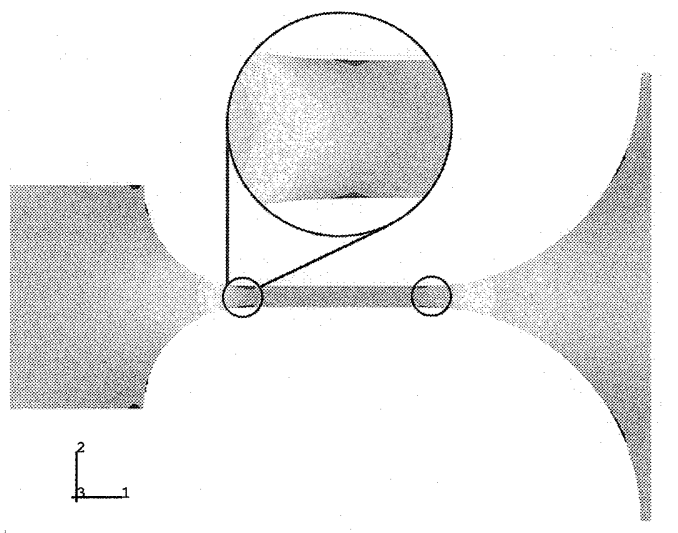

The fracture was brittle, as expected, and in most of the cases occurred at the ends of the test section as indicated by the finite element analysis of the specimen geometry shown in figure 3. The tensile stress assumes its highest value at the circled points (figure 3, right), differing by $4 \%$ from the central portion of the specimen. For specimens that were exposed to HF for longer times exceeding 15 minutes of exposure, the fracture occurred at random locations in the test section indicating that HF induced roughness in the form of scattered micronotches created sites for fracture onset.

\begin{tabular}{|l|c|}
\hline \multicolumn{2}{|c|}{ Table 1. Tensile test results } \\
\hline HF release time (minutes) & 13 \\
\hline Young's modulus (GPa) & $\mathbf{1 6 5 . 7} \pm \mathbf{4 . 7}$ \\
\hline Fracture Strength (GPa) & $\mathbf{1 . 0 0} \pm \mathbf{0 . 1}$ \\
\hline Friction Coefficient & $\mathbf{0 . 0 7} \mathbf{- 0 . 1}$ \\
\hline
\end{tabular}

The specimens were provided for "Round-Robin" experimental determination of physical properties of polysilicon specimens at the micron scale. ${ }^{5}$ All round-robin specimens were manufactured during the same run by Cronos. The results of this work agree well with results obtained by two of the other Round Robin participants. ${ }^{5-6}$ 
Although the aforementioned results were obtained using the closed loop inchworm actuator system to obtain the displacements, the strains of the surface of the specimen are currently measured using AFM records of deformed and undeformed specimen configurations. This is the final development stage of the described method. Figure 4 illustrates two AFM records of a deforming specimen. The first picture is the monitored area before the induced deformation i.e. at $0 \mathrm{~N}$ load while the second figure is captured during the deformation step of $0.06 \mathrm{~N}$ of axial load applied in the vertical direction of the figures shown. First point-to-point measurements provided a value of $155 \mathrm{GPa}$ for the elastic constant, which agrees well with the modulus obtained using the inchworm close loop indications. The investigation of the details for the application of the DIC method is currently in progress. Those include the identification of a necessarily large correlation domain, which is however sufficiently small from strain resolution point of view. Another consideration is the noise reduction to a degree that correlation errors are minimized and the efficiency of the method in detecting actual displacements is improved. At this point it is important to delineate the importance and the potential of the new method that can measure not only the elastic constants directly on the film, but also possesses the capability to monitor the deformation fields around notches and cracks with incomparable accuracy in the measurement of small displacements and provide enough information about the film microstructure (grain size, surface roughness) to correlate the measured properties with the physical condition of the test samples. As shown in figure 4, the surface topography is a complete record of the aforementioned data.


Figure 4. Left: Undeformed configuration with no applied load. Right: Deformed configuration with $0.06 \mathrm{~N}$ applied load.

\section{DISCUSSION}

A comparison of the acquired data using the new tensile test grip and the electrostatic method shows excellent linearity without slip and makes the use of AFM considerably easier. The absence of surface developed charges makes the use of a probe microscope possible in the regime of small interaction forces between the film and the AFM probe.

The value of the fracture strength of the MUMPs21 specimens reported in this work is considerably higher than that measured for MUMPs19 specimens in previous work ${ }^{6}$. It has been demonstrated that the low strength of specimens tested from MUMPs19 ( $\sim 0.3 \mathrm{GPa}$ ) is due to the long exposure of the latter ( $>20 \mathrm{~min}$.) to HF (to release them) when compared to the time used for the MUMPs21 specimens. Accordingly, the strength of the latter is lower than that which reported by other researchers and can be directly attributed to the fact that the etching time was longer than that they have stated. Further study, using an AFM, shows that the surface roughness is a strong function of specimen exposure to HF. An increase of HF exposure results in an exponential increase of the RMS surface roughness. Long exposure (15-20 min) leads to the appearance of deep random depressions. These deep surface grooves act as micro-notches that initiate fracture, and occur at the grain boundaries that are etched more readily and faster than the grains themselves. Analogous results have been reported in the literature for various treatments with hydrofluoric acid that resulted in variations in Young's modulus and failure strength. ${ }^{17}$ In addition, AFM data also show an influence of the grain structure on the character of crack propagation and a 
preference for intergranular fracture. While other researchers have reported transgranular fracture ${ }^{18}$, our AFM/SEM study has indicated an intergranular fracture type for our specimens. This represented an effect of the etching process on the grain boundary strength. SEM observations have also shown that the release-induced roughness is strongly dependent on residual film stresses. Films with large lateral dimensions displayed variable roughness across their width demonstrating increasing roughness away from the free edges of the film. This was due to the fact that although different areas of the top surface of the film were exposed to HF for the same time they were subject to the residual stresses for variable time as the release process progressed.

X-ray diffraction measurements ${ }^{19}$ revealed that layers with compressive residual stresses are strongly dominated by (110) orientation since the grains from LPCVD grow faster in the $<110>$ crystallographic directions, displaying a columnar structure. ${ }^{8,19}$ The Young's modulus of an isotropic polycrystalline solid can be calculated ${ }^{20}$ by averaging the modulus value of all crystallographic directions on a specific plane. Two models have been proposed: an isostrain, or Voigt ${ }^{21}$ model, for the upper bound, and an isostress, or Reuss ${ }^{22}$ model, for the lower bound, and the experimental value should lie within the VoigtReuss bounds. Using handbook values ${ }^{23}$ for the stiffness constants for $<110>$ texture, the modulus bounds are: $160<\mathrm{E}<166$ $\mathrm{GPa}$. Taking into account the preferred $<110>$ texture, as mentioned above, for LPCVD deposited films, our measured values agree well with the theoretical estimates. The columnar structure of the film, in conjunction with the applied force direction (action in series), would tend to yield modulus values close to the lower bound. However, the existence of other textures in smaller percentage, such as $<111>$ that is characterized by a modulus invariance with respect to the crystallographic direction $^{23}$ and a value of $168.9 \mathrm{GPa}$, and the deviations from the columnar structure, can lead to measurements outside the aforementioned bounds. Young's modulus depends on the interatomic distortions and it is not affected by the grain size of polycrystalline materials. But when the dimension of the specimen is small in comparison to the grain size, the mechanical properties are dominated by the grain orientations. ${ }^{23}$ In addition, the films under study were phosphorus-doped. Doping processes change the interatomic distance and thus the value of Young's modulus can be affected but by a few percent.

Accurate thickness measurements are important in order to evaluate mechanical properties. The uncertainty of the thickness of the film is increased by the fact that the surface displays roughness due to the exposed grains. A (two-dimensional) finite element program (ABAQUS) has been exercised ${ }^{6}$ to investigate the influence of surface roughness (bumps) as observed in the micro-manufactured specimens. The effect of the surface roughness on data accuracy is stronger in the case of bending tests, reaching values of almost $10 \%$ for small grain diameters and high roughness amplitudes. Taking into account the spatial roughness frequency, the error for our tensile test due to the existing roughness is on the order of $1.5 \%$. In the FEM model of the specimen geometry the only assumed value in the modulus calculation was Poisson's ratio $v$. The modulus value presented in table 1 was obtained using the experimentally available ${ }^{7}$ Poisson's ratio value of $v=0.22$. In theory, Poisson's ratio ranges ${ }^{23}$ between $0.18<v<0.28$ for the most common crystallographic directions of polysilicon. For this range, the value of Young's modulus, calculated by the FEM model, ranges in the interval $165.65<\mathrm{E}<165.8 \mathrm{GPa}$. Thus, the influence of this parameter is minimal for our work.

\section{SUMMARY}

A new and improved tensile tester has been devised. The new method incorporates a UV adhesive grip and is superior to the previously employed electrostatic grip, especially when an AFM is used to measure displacement fields. This improvement makes possible the test of hard, non-linear, or non-conductive materials with high rate of successful tests. By this method, the values of Young's modulus and fracture strength by means of force-displacement response of small-scale polycrystalline specimens were recorded. After adjustments to force-deformation data to allow for the compliance of the load cell, and a numerical evaluation of the deformed geometry, the material modulus was obtained. The results agree well with values obtained by the other researchers and by the bounds imposed by theory. Currently, surface measurements using an AFM are pursued and by use of the DIC method the strain fields are obtained with sub-pixel resolution. The AFM records provide information that addresses additional requirements of the deformation fields which are related to the physical structure of the film and provide input on the characteristic lengths of the grain size and surface roughness. Those length scales are important when specimens with dimensions of similar order of magnitude to the grain size are used for testing. Moreover, the surface roughness has been identified as an important parameter that depends on the exposure to HF acid during wet release and affects the tensile strength of the film. 


\section{ACKNOWLEDGMENTS}

The authors gratefully acknowledge the support by the Airforce Office of Scientific Research (Round Robin Program) through grant F49629-97-1-0324, under the direction of Major Brian Sanders and grant F49620-99-1-0091, under Drs. T. Hahn and D. Segalman.

\section{REFERENCES}

1. R. Howe, B. Boser and A. Pisano, "Polysilicon Integrated Microsystems: Technologies and Applications", Sensors and Actuators A, 56, pp. 167-177, 1996.

2. W. Lang, "Reflections on the Future Microsystems", Sensors and Actuators A, 72, pp. 1-15, 1999.

3. N. Yarzdi, F. Ayazi and K. Najafi, "Micromachined Inertial Sensors", Proceedings of the IEEE, 86 (8), pp. 1640-1659, 1998.

4. H. Fujita, "Microactuators and Micromachines", Proceedings of the IEEE, 86 (8), pp. 1721-1732, 1998.

5. W.N. Sharpe, Jr. S. Brown, G.C. Johnson, and W.G. Knauss, "Round Robin Tests of Modulus and Strength of Polysilicon", MRS Proceedings, 518, pp. 57-65, 1998.

6. I. Chasiotis W.G. Knauss, "Mechanical Evaluation of Polysilicon Properties by Means of Probe Microscopy", Proceedings of SPIE, 3512, pp. 66-75, Santa Clara, 1998.

7. W.N. Sharpe, K.T. Turner, and R.L. Edwards, "Tensile Testing of Polysilicon", J. of Experimental Mechanics, 39, (3), pp. 162-170, 1999.

8. S. Greek, F. Ericson, S. Johansson and J. Schweitz, "In Situ Tensile Strength Measurement and Weibull Analysis of Thick Film and Thin Film Micromachined Polysilicon Structures", Thin Solid Films, 292, pp. 247-254, 1997.

9. J. Koskinen, J.E. Steinwall, R. Soave and H.H. Johnson, "Microtensile Testing of Free-Standing Polysilicon Fibers of Various Grain Sizes", J. Micromech. Microeng., 3, pp. 13-17, 1993.

10. T. Tsuchiya, O. Tabata, J. Sakata and Y. Taga, "Tensile Testing of Polycrystalline Silicon Thin Films Using Electrostatic Force Grip", T.IEE Japan, 116-E (10), pp. 441-446, 1996.

11. D. Maier-Schneider, J. Malbach and E. Obermeier, "Variations in Young's Modulus Intrinsic Stress of LPCVD Polysilicon due to High-Temperature Annealing", J. Micromech. Microeng., 5, pp.121-124, 1995.

12. F.A. Breton and W.G. Knauss, "Error Limitations in the Determination of Mechanical Properties of Thin Films", Journal of Reinforced Plastic and Composites, 16 (1), 1997.

13. G. Vendroux and W.G. Knauss, "Submicron Deformation Field Measurements II: Improved Digital Image Correlation", Experimental Mechanics, 38 (2), pp. 86-92, 1998.

14. M.A. Sutton, M. Cheng, W.H. Peters, Y.J. Chao and S.R. McNeil, "Application of an Optimized Digital Image Correlation Method to Planar Deformation Analysis," Image Vision Computing, 4 (3), pp. 143-150, 1986.

15. R. Maboudian and R.T. Howe, "Critical Review: Adhesion in Surface Micromechanical Structures", J. Vac. Sci. Technol. $B, 15$ (1), pp. 1-20, 1997.

16. N. Tas, T. Sonnenberg, H. Jansen, R. Legtenberg and M. Elwenspoek, "Stiction in Surface Micromachining", J. Micromech. Microeng., 6, pp. 385-397, 1997.

17. J.A Walker, K.J. Gabriel and M. Mehregany, "Mechanical Integrity of Polysilicon Films Exposed to Hydrofluoric Acid Solutions", Journal of Electronic Materials, 20 (9), pp. 665-670, 1991.

18. R. Ballarini, R.L. Mullen and A.H. Heuer, "The Fracture Toughness of Polysilicon Microdevices: A First Report", $J$. Mater. Res., 12 (4), pp. 915-922, 1997.

19. P. Lange, M. Kirsten and W. Riethmuller, "Thick Polycrystalline Silicon for Surface Micro-Mechanical Applications: Deposition, Structuring and Mechanical Characterization", Sensors and Actuators A, 54, pp. 674-678, 1996.

20. R. Hill, "The Elastic Behavior of a Crystalline Aggregate", Proc. Phys. Soc. London, Sect. A, 65, pp. 349-354, 1952.

21. W. Voigt, "Lehrbook der Kristallphysik", $2^{\text {nd }}$ Edition, pp. 962, Teubner, Leipsig, 1928.

22. A. Reuss, "Berechnung der Fliessgrenze von Mischkristallen auf Grund der Plastizitaettsbediengung fuer EinKristalle", Z. Angew. Math. Mech., 9 (1), pp. 49-58, 1929.

23. W.A. Brantley, "Calculated Elastic Constants for Stress Problems Associated with Semiconductor Devices", J. Appl. Phys., 44 (1), pp. 534-535, 1973. 